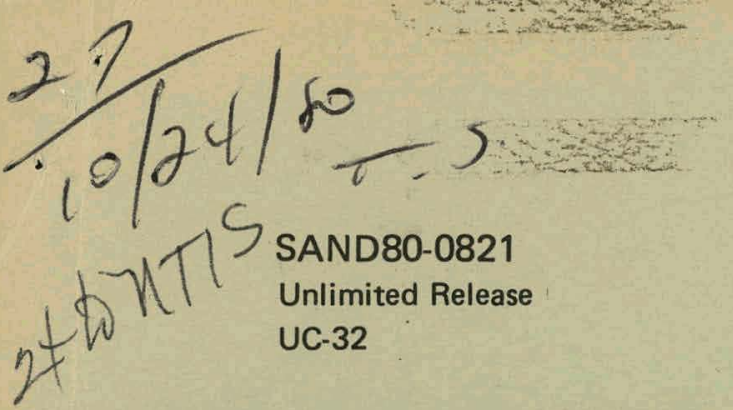

\title{
An Overview of a Minicomputer Network
}

\section{MASTER}

Michael O. Vahle, Lawrence F. Tolendino

Prepared by Sandia National Laboratories, Albuquerque, New Mexico 87185

and Livermore, California 94550 for the United States Department of Energy

under Contract DE-AC04-76DP00789

Printed August 1980

\section{Sandia National Laboratories}




\section{DISCLAIMER}

This report was prepared as an account of work sponsored by an agency of the United States Government. Neither the United States Government nor any agency Thereof, nor any of their employees, makes any warranty, express or implied, or assumes any legal liability or responsibility for the accuracy, completeness, or usefulness of any information, apparatus, product, or process disclosed, or represents that its use would not infringe privately owned rights. Reference herein to any specific commercial product, process, or service by trade name, trademark, manufacturer, or otherwise does not necessarily constitute or imply its endorsement, recommendation, or favoring by the United States Government or any agency thereof. The views and opinions of authors expressed herein do not necessarily state or reflect those of the United States Government or any agency thereof. 


\section{DISCLAIMER}

Portions of this document may be illegible in electronic image products. Images are produced from the best available original document. 
Issued by Sandia National Laboratories, operated for the United States Department of Corporation.

NOTICE: This report was prepared as an account of work sponsored by an agency of the United States Government. Neither the United States Government nor any agency thereof, nor any of their employees, nor any of their contractors, subcontractors, or their employees, makes any warranty, express or implied, or assumes any legal liability or pronst or pror the accuracy, completeness, or usefulness of any information, apparatus, proned rights. Reference herein to any specific by trade name, trademark, manufacturer, or otc comise imply its endorsement, recommendation or favoring by the United States Government any agency thereof or any of their contractors or subcontractors. The views and opinion, expressed herein do not necessarily state or reflect those of views and opinions Government, any agency thereof or any of their contractors or subcontractors.

Printed in the United States of America

$$
\text { Availahla from }
$$

National Technical Information Service

U.S. Department of Commerce

5285 Port Royal Road

Springfield, VA 22161

NTIS price codes

Printed copy: $\$ 6.00$

Microfiche copy: A01 


\section{PAGES 1 to 2 WERE INTENTIONALLY LEFT BLANK}


SAND80-0821

Unlimited Release

Printed August 1980
Distribution

Category UC-32

AN OVERVIEW OF A MINICOMPUTER NETWORK

\author{
Michae 1 0. Vahle \\ Lawrence $F$. Tolendino \\ Computer Communications Design Division 2648 \\ Sandia National Laboratories \\ Albuquerque, NM 87185
}

\begin{abstract}
A computer network was developed to support minicomputers used at a number of locations within Sandia National Laboratories. This report describes the control strategies, capabilities, and design philosophies of the minicomputer network.
\end{abstract}




\section{ACKNOWLEDGMENT}

The project described in this paper is the result of the joint efforts of the members of Division 2648, Sandia National Laboratories, Albuquerque, NM. Those making significant contributions include Steven Gossage, Lynda Pierson, Donald Schroeder, Mark Thompson, Terry Unkelhaeuser, David Whelan and the authors. 
CONTENTS

Introduction

Page

Network Philosophy

Network File Description

Network Management

Network Message Submission

12

Network Message Retrieval

16

Central Site Communication

19

APPENDIX -- A Queuing Scheme

21

25 


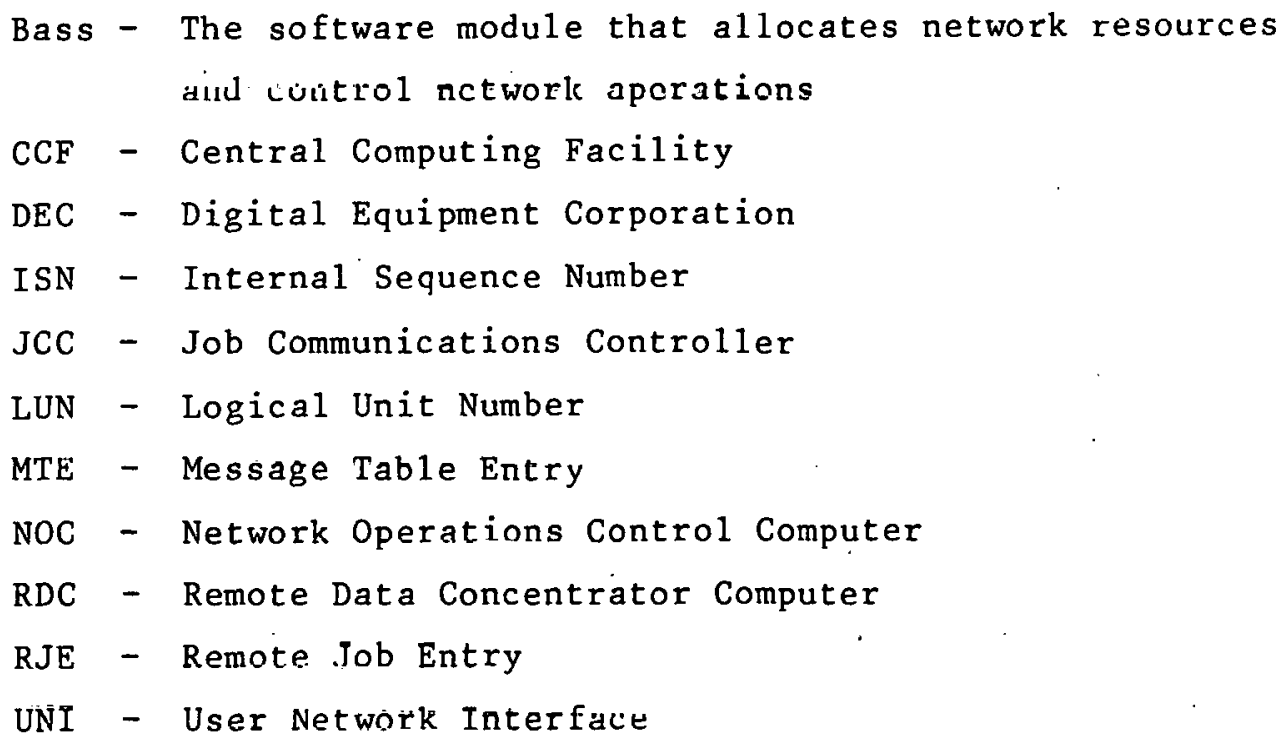


AN OVERVIEW OF A MINICOMPUTER NETWORK

Introduction

This report describes a computer network implemented to support the needs of the growing number of minicomputers at Sandia Laboratories. These minicomputers are of various types and are spread over a considerable area. Their needs are split between the ability to transmit data to the Central Computing Facility, and the ability to share data with other minicomputers. To satisfy these needs, a computer communications network was designed. The sequence of events, network control strategies, and network data structures used in this network are detailed.

\section{Network Philosophy}

The network is structured for store and forward message-switching (Figure 1). Messages entering the network are accumulated in their entirety at the central network node before any action to deliver them is taken. This structure, while introducing long delays, effectively decouples the source from the destination. This decoupling is of great benefit to the typical minicomputer user whose system is not capable of concurreul activities. An analogy with the postal service can be drawn: messages are submitted from various locations, sorted at a central location, and distributed to individual's mail boxes. In our case, the messages are computer files and once they are submitted to the postal service (the minicomputer network), responsibility for delivery rests with the network. The messages (files) remain within the network until the addressee requests delivery. Once the total message is delivered, the network relinquishes all responsibility. As a corollary to this network philosophy, real-time computer networking is ruled out. 


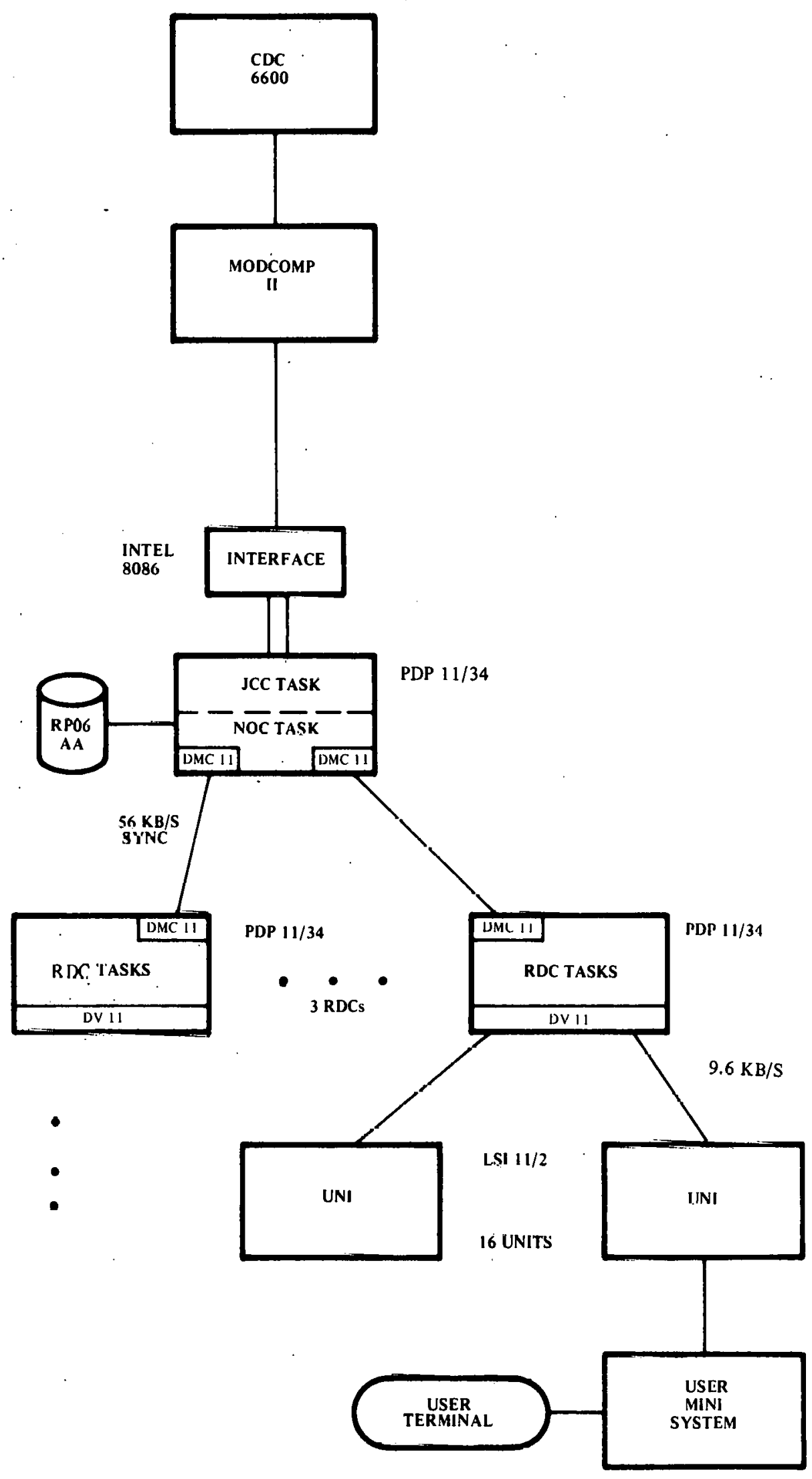

Figure 1. Network Structure 
A hierarchical network structure was chosen consisting of a central network node (NOC), a set of data concentrators ( $R D C$ ), and microprocessor based user network interfaces (UNI). The typical message flow is from user computer through a UNI and RDC to the NOC. At the NOC, the file is stored until requested by the destination node. Once requested, the file is sent from the NOC through an RDC and UNI to the destination computer. The NOC and RDC are implemented with DEC PDP 11/34s. The UNI is implemented with DEC LSI 11s. These choices allowed for the use of the DEC operating system RSX-11 and the interprocessor communication package DECNET. The network utilizes DECNET's intertask communication, remote file access, and downline loading capabilities.

The network implementation limits the number of simultaneous active users. The network design can accept up to 48 users, however only 16 can be active at any one time (transferring files) because of the following 1 imitations :

1. DECNET supports up to 20 simultaneous file accesses at a single node. The network software requires up to 4 of these at the NOC leaving only 16 for user computers.

2. Since DECNET remote file accesses cannot be overlapped in any one task, it is necessary to have individual RDC tasks for each active UNI. This constraint, in conjunction with PDP $11 / 34$ memory size, limits the number of active users per RDC to 6 .

The network supports 1 ong and short messages which are used for differenl purposes. Long messages are used to transfer files from computer to computer. Short messages are used to pass a limited amount of information between user computers, UNIs, the Central Computing Facility (CCF), and the NOC. These two types of messages are handled independently throughout the network, but both are serviced on a first-in, first-out. basis. For example, the user can choose to receive either a short or long message, but he must receive the first (oldest) message of that type.

In order for minicomputers to gain CCF access, a remote job entry (RJE) protocol has to be used. To implement this protocol; a special node 
called the job communications controller (JCC) was added to the network. The JCC receives short and long messages from the network and submits them in proper format to the CCF. Additionally, the JCC retrieves messages from the CCF and transmits them to the NOC for later acceptance by a network node.

The network enables the user to:

1. Submit files (long messages) to the network for delivery to a specified node. This node can be either a minicomputer connected to the network or the CCF.

2. Retrieve files that are waiting at the NOC for delivery to uspr. These files originated from a minicomputer cumnected to the network or from a CCF job.

3. Request network status to be displayed at the iuitiating console. This status message consists of an indication of network activity, resource availability, and the status of files in trausit to and from the requesting node.

4. Send short informational messages to any specified network mode. These nodes consist of any connected minicomputer, the CCF, or the Nmmonsole. For example, these messages can be used to trace job execution at the CCF.

5. Receive short messages waiting at the NOC for delivery to the user. The messages can originate at any minicomputer connected to the network, the CCF, or the NOC console. For example, this message could be the reply from the CCF to a request for job status.

Al1 of the above functions can be executed from the minicomputer console by using a utility program in the user minicomputer. In addition, functions 3 through 5 can be performed from the console attached to the UNI. This UNI function allows the user to perform many of the network activities without dedicating his minicomputer to the task, however, the minicomputer is required whenever mass atorage is needed, spccifically to transfer files. The replication of these functions in both the UNI and user minicomputer was included to permit decoupling the user minicomputer from the network. 
A message residing on the NOC file storage media consists of a number of 512-byte logical blocks. These blocks consist of a 6-byte network message header and up to 506 bytes of user data. The user data consists of mini-computer records preceded by a byte count (two bytes) indicating the size of the following record. Additionally, to provide data transparency and to indicate nonambiguous CCF record marks, special records may be imbedded in the user's data. These special records are inserted and removed by the utility program running in the user minicomputer or the JCC node. Specifically, records indicating the CCF record marks, EOF and EOR, represented in the network by a record containing the appropriate ASCII characters are protected. These records aré always preceded by a record containing the character string DLE. Since the string DLE delineates a CCF record mark, a record consisting of DLE must be inserted in front of any occurrence of an identical record in the user data. This record-stuffing concept is an extension of the byte-stuffing technique used in some byteoriented proto-cols.

The six bytes of network header on files transmitted from a minicomputer are generated within the UNI from information supplied by the UNI console or the user utility program. Similarly, the JCC generates a network header for files being returned from the CCF from information contained in the banner page. For short messages, the JCC builds the header from network header information contained in the file to which it is responding. The meanings of the six network header bytes are:

1. The first byte indicates the type of message.

$$
\begin{aligned}
& D=\text { a long message } \\
& S=\text { a request for network status } \\
& M=\text { a shnrt message }
\end{aligned}
$$

2. The second byte indicates the originating node for the message. There is a unique number for each node in the network ranging from 1 to 50 . For instance, the CCF is designated as node 49. 
3. Bytes three and four contain an internal sequence number (ISN) in the range of 1 to 32 000. This number affords a unique identification to each message within the network. The number is assigned by the NOC when resources for this message are allocated.

4. Byte five indicates the destination using the same numbering convention as source identification. This number is used by the NOC to perform message routing.

5. Byte six is the last part indicator. It is zero in all parts except the last where it is one.

Network Management

A naming convention was adopted that names the messagc according to the resuurces allocated to keep track of network messages. For example, when a message is submitted to the network, a message table entry (MTE), is assigned to the message. MTEs are in the range of 1 to 100. If MTE 5 is assigned to the message, the file name for the message on the NOC disk will be M005. The M'E is a pointer into a table that contains the parameters needed to process the message. Parameters included within the table are: originator of the message, uumber of meocago dostinatinns, and the ISN identifying the message. Along with the MTE tabie, there is a logic unit table which is indexed by logic unit number in the range of 1 Lo 16 . When a message is being transferred, a DECNET 1 ink is established on 1 of 16 logic links. Ihe lugic link indexco the logir unit table where the MTE and message descriptors are stored. Whenever an action such as transmission or reception complete occurs, the network reports the occurrence through a DECNET disconnect on the logic unit associated with the estab1 ished 1 ink. The information from the disconnect is stored in the logic unit table along with the MTE number until the BOSS, the NOC control program, can process it. During a transfer, a message is assuciated with a logic unit number. When the file is being stored in the NOC, the MTE identifying the message is stored in a queue assigned to the destination. In both cases, the information to process the message remuins in memory and is queued so that the BOSS, when requested, can handle the message without accessing the disk. 
At network initialization, the BOSS examines the network storage disk for filenames of the type Mxxx. Upon encountering such files, the appropriate MTEs are created and entered in the common network queue for subsequent routing. Additionally, network parameters stored on safe media are read. These parameters include the current configuration, the current network $\log$ position, and the most recently assigned ISN. This mechanism provides the means of recovering from the last known operating environment. Additionally, all messages, once accepted by the network, can be delivered after a network initialization.

The program BOSS controls the NOC and its console. When a command has been entered on the NOC console, the BOSS determines if it is a network command by comparing it to a list of known commands. If a match is found, the BOSS processes the command. If no match is found, the command 1 ine is passed to the program CRAMIT using the RSX-11 send/receive directives and subsequently submitted to the RSX-11 executive. Output generated in response to a network command is handled in one of two ways: either the output is buffered and queued to the terminal using an RSX-11 QIO, or an MTE entry is generated and queued to the routine OPMSG. In either case, the BOSS's execution is not suspended awaiting I/O completion.

The operator can execute the following network commands:

1. STAT = generate a network status report on the NOC console. This message contains the date, the time, and the number of free network resources.

2. LOGT = declare the NOC console as the system log device. This causes all network transactions to be recorded on the NOC console as they are being recorded on the network log disk.

3. $L O G D=\log$ to network $\log$ disk (default state).

4. LIST $x x(q)=1$ ist the ISNs in user $x^{\prime}$ 's queue on the NOC console. If $(q)$ is not specified, the long message queue is listed. If $(q)$ is specified as an $S$, then the short message queue is displayed. 
5. PURG $x x$ yyyyy $(q)=$ the file identified by ISN yyyyy is removed from user's xx queue. The default for this command is the long message queue, but can be overridden by specifying $(q)$ as an $S$, indicating that the message is in the short message queue.

6. CFIG = display on the NOC console the current assigned nodes and owning organizations.

7. SHUT $=$ bring the network to a graceful shutdown. While the network is shutting down, no new message transfers are begun and those in progress are allowed to complete. Shutdown is accomplished when all logical units are inactive and the BOSS terminates and removes itself from the system.

8. MAIL $x x$ text $=$ place a llessage eonsioting of text in user $x x^{\prime} s$ short message queue.

Once the network is initialized, the network control task BOSS, is normally inactive, awaiting notification from the operating system that a significant event has occurred. When a significant event occurs, BOSS determines if action is necessary. Action is necessary if a network command has been entered, a user request to transmit a message has been received, a user request to receive a message has been received, or a network message transfer has been completed.

A network requesL LU Liansmit a mcooage is generated by a DECNET request to connect. The request contains pertinent information such as the destination node. The BOSS validates the destination node number and insures that an MTE and LUN are available and that a network shutdown is not in progress. If either of these checks fail, a DECNET reject is returned containing the reason for rejection. If all checks succeed, the connecioin is accepted and an MTE, an ISN, and destination Identificalion is returned. The BOSS initializes the necessary tables for this tranbfer and takes no further action until notified through a DECNET disconnect that the rransfer is complere.

Similarly, a network request to retrieve a message is generated by a DECNET request to connect. In this case, the request contains the requesting node's identification number and the desired queue. The node number is 
verified, the queue is checked to see that it is not empty, and the appro priate resources checked. If any of these checks fail or a network shutdown is in progress, a rejection is sent containing the reason. If all checks succeed, the first MTE in the queue is returned in the accept. The appropriate table entries are made, and the BOSS takes no further action'. until notified by a DECNET disconnect that the transfer is complete.

If a DECNET disconnect is received, the information contained in the disconnect is evaluated. When the disconnect indicates that the message transfer was completed successfully, the file will either be queued to the: appropriate destination or deleted from the NOC disk, depending on whethe the LUN was assigned to a submit or to a retrieve function. If the disconnect indicates an unsuccessful message transfer, the message is requeued to the destination if it was being retrieved, or it is deleted if it was being submitted. In all cases, the appropriate dedicated network resources are released.

Whenever the BOSS must take action on a file transfer, the action is. logged. These actions include accepting or dropping responsibility for network messages, routing network messages, and generating network status reports. The logging is accomplished by queueing an MTE containing the pertinent information about the transaction. This MTE is later processed by routines in BOSS resulting in an entry on the $10 g$ device. The $10 \mathrm{~g}$ device normally contains the last 4000 entries stored in circular buffer.

A message placed in the NOCs short message queue is eventually printed on the NOC console. This is the method the JCC uses to 109 abnormal activity on the CCF link.

Two programs, BANISH and REDEEMER, provide the capability to remove. and restore messages in specified queues. BANISH, functioning as a network node, retrieves messages from the user's queue and places them on magnetici. tape. The messages are stored on tape in the same format they were stored on the NOC disk. REDEEMER can read any tape created by BANISH and submit : the messages on that tape to the network. The two programs, under operator: control, work in concert to manage critical network resources. 
Techniques used to maintain the NOC queue structures are described in the Appendix. The queues are tirst-in, first-uul linked listo. Entries ran be made at the top or bottom of these queues; however, entries can only be removed from the top of the queue.

The current network ISN, network node identifications, and $10 g$ position are maintained on the network $\log$ disk. This information is accessed by BOSS whenever the network is initialized. To update this information, a program called TABLES is executed which contains the current network configuration. It is possible to change the configuration by editing TABLES, executing a network shutdown, running the program IABLES, and restarting the network.

Network Message Submission (Figure 2)

In order for a user to submit a message to any network node, the network utility program must be executing. The utility program prompts for file names and the destination. Afcer receiving this information, the utility program connects to its UNI by sending a request to transuit containing the desired destination. At this time, the utility program starts a timer. Should this timer expire before a response from the UNI is received, the urflity plugitan notifico tho user and terminates. When the UNI rcccives the request to transmit, the program sends a DECNET connect request to the RDC program SCHEDULER. If no RDC service programs are free, the SCHEDULER returns a zero in its DECNET reject. In this case, the UNI. sends an error indication to the utility which notifies the user and terminates. If a service program is free, the STCHEDULEk includes a designator identifying the free service program in its DECNET reject. The UN then connects to the designated RDC service program passing the destination and a request to transmit. 


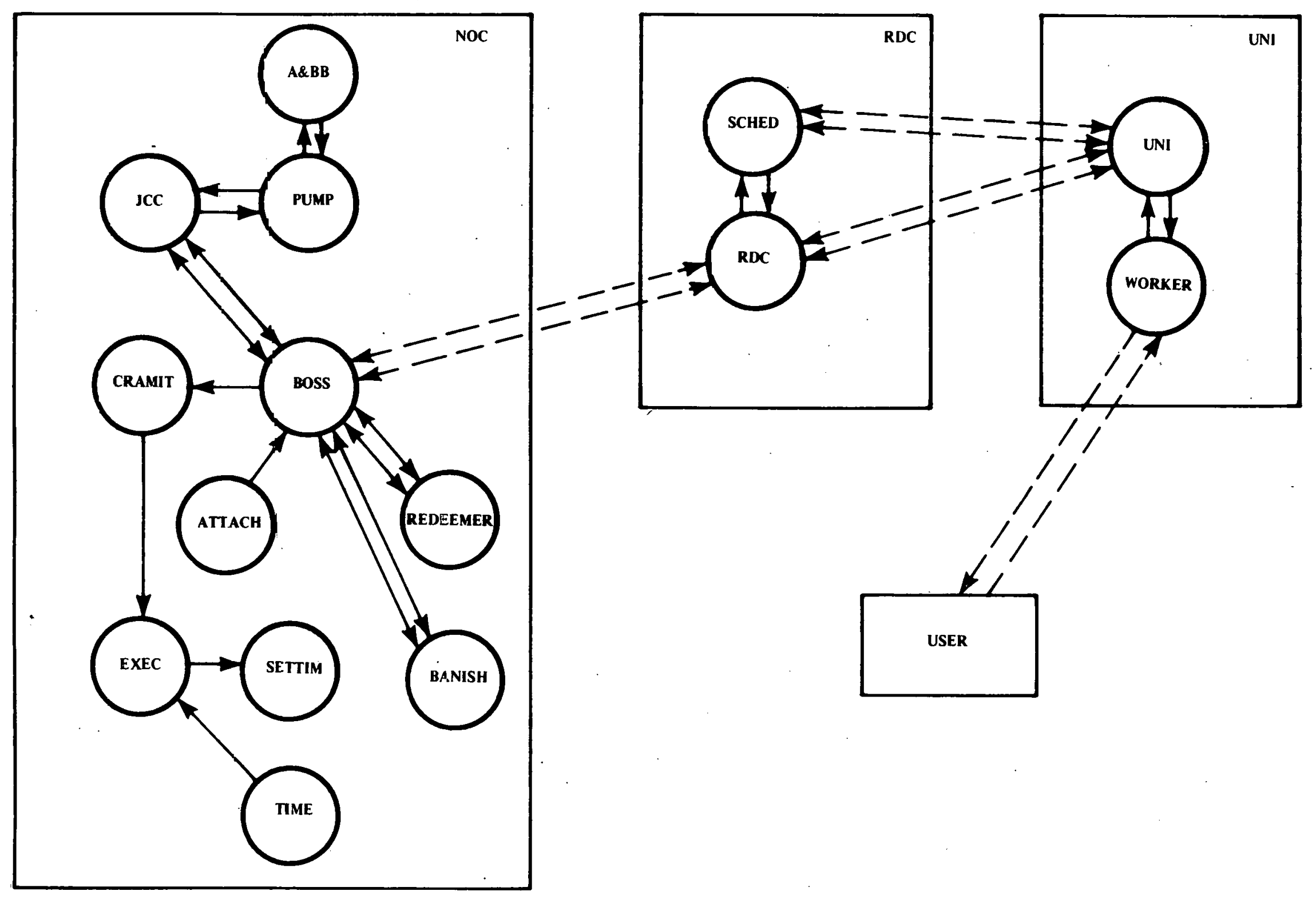

Figure 2. Network Interactions 
At this point, the RDC service routine issues a DECNET connect request to the NOC control program, BOSS. The connect request contains a iequcot to transmit and the destination. The BOSS either accepts or rejects the request as outlined in the file management section. If the request is rejected, a reason is returned. This reason is then passed in the service program's rejection to the UNI which passes it to the utility program. The utility program notifies the user and terminates. If the BOSS accepts the connection, an MTE, an ISN, and destination identification is returned to the service program within the connection accept. The RDC service routine uses the MTE to build the file name used for this transfer and passes the rest of the information to the UNI in its connection accept. The UNI uses the ISN to build the network header and pasger ull the information to the utility program in its response. The utility program then displays this information to the user. At this point, Llie connection is established and data transfer can begin.

The utility program begins reading the indicated.files a record at a time. The records are placed in a 506-byte buffer preceded by the record byte count. When 506 bytes have been accumulated, or the last record in the files has been processed, the utility program attaches the byte count of the data and a last part indicator. The buffer is sent to the UNI and a timer started. If the timer expires before the UNI responds, the utility program notifies the user and rerminates. The UNI lakes the data, attaches the proper network header, transmits the packet to the designated RDC service program, and acknowledges the utility program. The UNI chen starts a timer waiting for the next transmission from the utility program. In the meantime, the service program writes the packet to the Nos diak using a. DECNET utility FAL. If the UNI timer explyes before the ncxt packet is received from the user utility program, the UNI sends a retransmission request to lie utility progr au and rcotarts the timer. This recovery technique will be tried three times before failure is reported. The above procedure continues until either the last part is processed or a fatal elior occuro. When the last part is transmitted by the utility program, the UNI refrains from acknowledging the utility program until the service routine informs the UNI that the last part has been written to disk, the file has been successfully closed, and a proper disconnect to the Boss has 
been executed. This information is passed in the disconnect breaking the link between the UNI and the service routine. The final acknowledgment to the utility program indicates that the file transfer is complete and that the network has accepted responsibility for the file.

If an error occurs during the file transfer and recovery techniques fail, the error is reported along with the failure type to the Boss by executing a DECNET disconnect on the link established at connection time. This error may be detected by the service program or the UNI. In the case where the error is detected by the service program, the service program disconnects from the UNI and the BOSS and indicates the reason for the disconnect. The UNI passes this information to the utility program so that the user can be notified. In the other case, the UNI notifies the utility program and disconnects from the service program which passes the disconnect on to the BOSS. In either case, the BOSS deletes the file referenced by this connection and frees all network resources supporting this transfer.

The above description also applies to short messages input from the user computer. Short messages can also be input from the UNI console. In this case, the references to the utility program are not applicable, and all necessary parameters are input from the UNI console.

\section{Network Message Retrieval}

To retrieve a message from the network, the user must execute the network access utility program in his minicomputer. The utility program prompts the user for the name the message is stored under. After these parameters are entered, the utility program requests the UNI to retrieve the firsl message in its queue and starts a timer. If the timer expires before the UNI responds to the request, the utility program notifies the user and terminates. The UNI, upon receiving the request, issues a DECNET connect to the RDC SCHEDULER program. If there are no free RDC service programs, the SCHEDULER returns a zero in its rejection. The UNI then rejects the utilities program request forcing the litility program to 
notify the user and terminate. When a free service program exists, the SCHEDULER rejects the connection returning a designator for the free service program. The UNI then connects to the free selvice program passing the queue number generated from the utility program request. The service program then issues a DECNET connect to the BOSS passing along the same information. If there are no files in the specified queue, insufficient network resources, or the network is shutting down, the connection is rejected and the appropriate reason is returned. The service program then passes the reason for rejection to the UNI and makes itself available to the SCHEDULER. The UNI in turn passes the reason on to the utility which notifies the user and terminates. If the BOSS accepts the connection, it returns the MTE of the first message in the specified queue. The service program then uses the MTE to build the file name and npens that file. If the file fails to open, the service routine disconnects from the Boss and rejects the UNI request. The UNI then notifies the utility program of the error, and the utility program notifies the user and terminates. Once the file is opened successfully, the service program accepts the UNIs connect request, the UNI acknowledges the utility program request and data transfer can begin.

When the connection is established, the service routine reads a $312-$ byte block frum the filc on the Nul: Alsk, using the DECNET remote file access utility FAL. This block is thea seit to the UAl. Thr ImT atring fhe 6-byte network header from the beglnniug of the blook, attarhes a last part indicator, and transmits the first half, to the utility program. The utility program acknowledges the first half and disassembles the block writing the individual records to the minicomputer file storage device. The records are delineated by imbedded byte counts. After receiving the utility program's acknowledgment, the second half of the block is transmitted by the UNI. Succeeding blocks are transferred in this manner until the last block is acknowledged by the utility program. Then the UNI disconnects from the service program indicating a successful file transfer. Subsequently, the service program disconnects from the BOSS with a similar indication. The BOSS then releases dedicated resources and deletes the file associated with the connection from the NOC disk. 
In case of a data transfer error, the logical link established at connect time is disconnected. The reason for the disconnect is passed and logged on the NOC log device. The message that was in transit is requeued to the top of the destination's queue. This disconnect procedure can be initiated by the UNI or the RDC service program. When a disconnect is initiated by the service program, a subsequent disconnect is sent to the UNI including the reason for disconnect. When the UNI detects the error, it issues a disconnect containing a reason to the service program. In both cases, it is the responsibility of the UNI to notify the utility program that an error has occurred, while it is the responsibility of the service program to notify the BOSS. The utility program closes any open files, notifies the user of the error, and terminates.

Short messages are retrieved in a similar manner when the request is initiated from the user's minicomputer console. However, in this case, a local file is not created, and the message is output on the console. Short messages can also be retrieved on the UNI console. AlI parameters are input from the UNI console, and the user minicomputer plays no role.

\section{Central Site Communication}

Communications between the network and the CCF are conducted by using existing RJE protocols. The link-level protocol is PUMP, and the highlevel protocol is BART. The node JCC implements these two protocols and interfaces the network to the CCF. The JCC is a hybrid network node incorporating many of the capabilities of an RDC server, a UNI, and a user. utility program. CCF jobs are submitted to the JCC's long message queue, while BART commands are sumbitted to the JCC's short message queue. The output generated from CCF jobs input by the JCC is routed to the user's long message queue, while the responses to BART commands are returned to the user's short message queue.

The JCC is normally in a polling mode, checking its two network queues for CCF input and the three CCF data streams for network input. The polling to the network is accomplished by issuing a DECNET connect to Llie 
ROSS first to the short message queue and then to the long message queue. Next, the three CCF output data streams are checked to see if any data packets have been received.

If the short message queue connection is accepted, the BOSS returns the first MTE in the queue. The JCC uses this to build a file name and opens that file using the DECNET utility FAL. The JCC reads the short message, closes the file, and disconnects from the BOSS. The BOSS deletes the message and frees all network resources. The JCC then submits the contents of this message to the TTY data stream and waits a set amount of time for a reply. It the CCF replies, or the JCC's Limei expires, a short message is sent to the requesting user. This message contains the reply to the BART command or a statement that a time-out occurred. The short message is submitted to the network by requesting a DECNEI connect to the BOSS. If the BOSS accepts the connection, an MTE is returned and the JCC writes the message to the NOC disk with the appropriate header. Any errors that occur are ignored.

When the connection for the long message queue is accepted, the Boss returns the MTE for the first message in the queue. The JCC builds the file name and opens the file using the DECNET urility FAL. The filst block of the message is read, and the first record in the block (the job control card) is modified. The originator's node number is inserted in the second and third position of the job name. This job name modflcation alluws the JCC to determine the destination for CCF output by reading the job name on the banner page. The records in this and subsequent blocks are input to the card reader data stream. When the last record is shipped, the file is closed and the link to the BOSS is disconnected. The BOSS then deletes the message from the network disk and frees all associated resources. The JCC then sends a short message to the user confirming job submission and associating a CCF job name to the message's ISN. If an error occurs during job submission, the link to the BOSS is disconnected. The BOSS logs the error, releases network resources, and returns the MTE to the top of the JCC's long message queue. 
When the first packet of CCF output on the line printer or plotter data streams is received, the JCC checks the job name to determine that the job is destined for a network node. If it is not, the output is returned to its queue, the data stream is turned off, and the file is diverted or evicted depending on type. If a legal node number is present in the job name, a connect is issued to the BOSS. If the connection is rejected, the file being delivered is returned to its CCF output queue. Otherwise, the BOSS's reply contains the MTE and ISN to be used. The JCC then accepts following records and writes them to the NOC disk using the DECNET utility FAL. The blocks are 512 bytes long and contain the network header. When the last block is written to disk, the JCC disconnects from the BOSS and closes the file. If any errors occur, the CCF output file is replaced in its queue, and a disconnect is sent to the BOSS indicating the error type. The BOSS deletes the file and frees its resources. 


\title{
APPENDIX
}

\author{
A Queuing Scheme
}

\section{Genera1}

The following documentation outlines a means of queuing varied jobs among many tasks within a common software system. The parameters needed to perform a task are passed in a common area called MTES. The MTES associated with a particular task are linked together and placed in a user queue. Each task is able to process these jobs by using subroutines that make the queues and linking transparent.

For example, suppose a line program has just accepted a message off of a line and has stored it on disk. The message must be passed to a distribution program where the routes will be decoded and a message generated to each addressee. To accomplish the transfer of information about the location, as well as any other necessary parameters, the 1 ine program would execute the following steps.

1. Request a spare MTE from the system pool

2. Put the necessary parameters into the MTE

3. Request the MTE to be put into the distributions program queue.

The distribution program would in turn perform the following steps. Eventually the MTE placed in its queue by the line program would be processed.

1. Request the next MTE in its queue

2. Use the information in the MTE to perform ito tasks

3. Return the MTE to the system pool

4. Prepare to process the next message. 
Details

Initially, the MTES are linked in a serial 1 ist. The first entry in each slot in MTES contains the index of the next slot in the 1 ist. The last slot in the list contains a zero in its first entry. Two pointers MNEXT and MLAST contain the indexes of the first and last slots in the 1 ist. When a user requests an MTE, the MTE associated with the index in MNEXT is passed to the user. Then MNEXT is updated from the first entry in . the slot given to the user. When a user returns an MTE, the associated index is placed into the first entry of the slot pointed to by MLAST. Then MLAST is updated to point to the MTE being returned.

The following diagrams demonstrate a request and recurn uf á MTE: MTES MTES
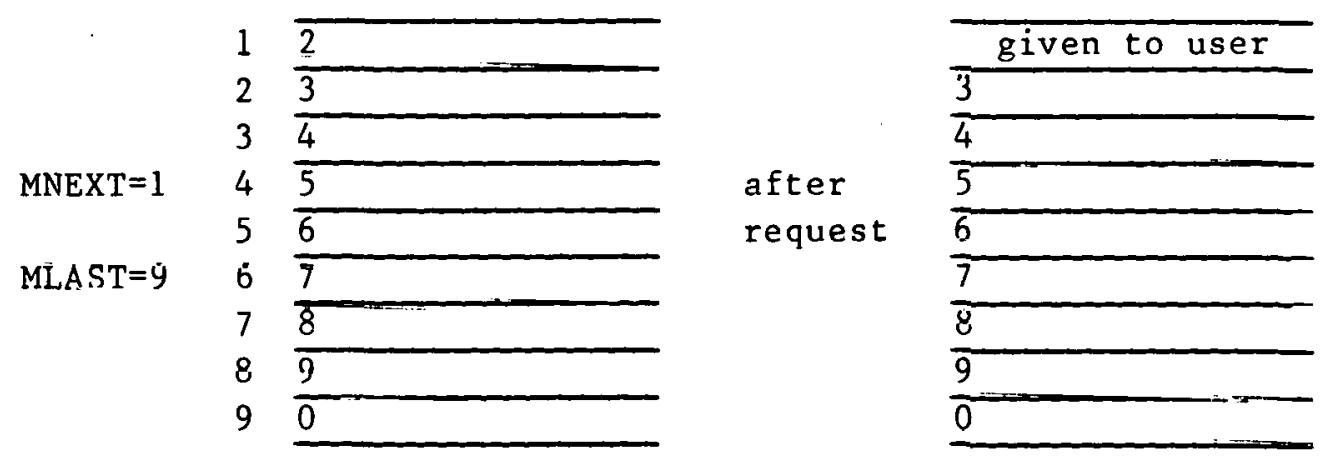

MNEXT $=2$

MLAST $=9$

\begin{tabular}{|c|c|c|}
\hline & 1 & users \\
\hline & 2 & $\overline{3}$ \\
\hline & 3 & 4 \\
\hline \multirow[t]{2}{*}{$M N E X T=2$} & 4 & 5 \\
\hline & 5 & 6 \\
\hline \multirow[t]{4}{*}{ MLAS'I' $=9$} & 6 & 7 \\
\hline & 7 & $\overline{8}$ \\
\hline & 8 & $\overline{9}$ \\
\hline & 9 & $\overline{0}$ \\
\hline
\end{tabular}

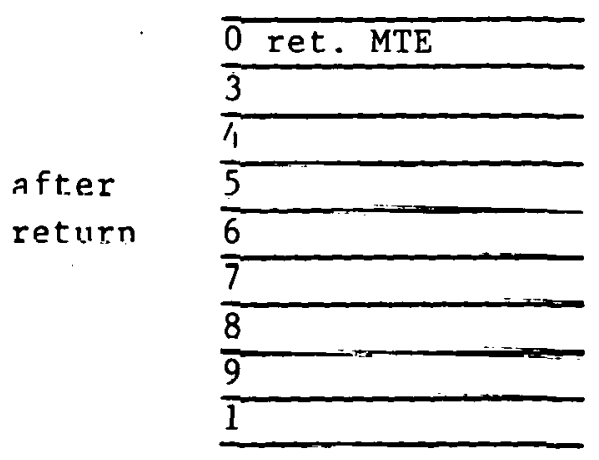

MNEXT $=2$

MLAST $=1$ 
A similar scheme is used on LINK. However, the pointers MNEXT and MLAST are replaced with LNEXT and LLAST, respectively. The above diagrams represent this scheme if the appropriate changes in nomenclature are kept in mind.

A. queue is a two-entry data structure that contains the indexes of the first and last links to be serviced. Each link specified contains the index of the next link in the list and the index of the MTE containing the job parameters. Consider the following diagram which depicts a queue called JOBQUEUE with three jobs scheduled for service (the indexes of the MTES in the QUEUE are $3,5,7$ ).

LINKS

JOBQUE
\begin{tabular}{cc} 
\\
\hline 3 & 6 \\
\hline first & last \\
link & link
\end{tabular}

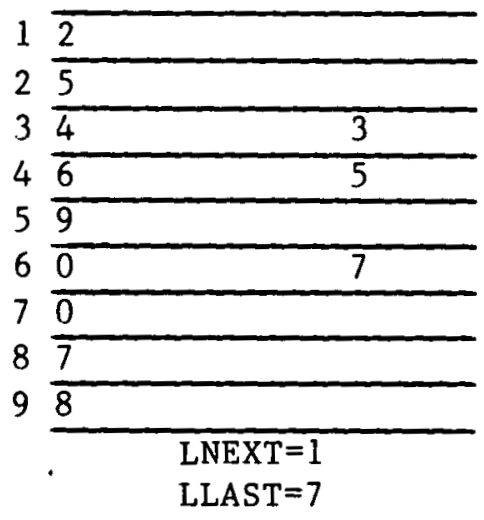

MTES

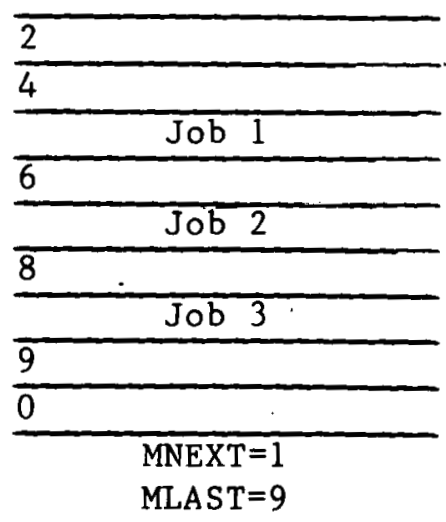

Now, if a user requested an entry be taken from the queue, the index in the first entry of JOBQUE would point to the associated link. This link contains the next link's index which updates the first entry in the queue. Also, it contains the index of the MTE which is passed to the user. The. freed LINK is then returned to the system pool. The following diagrams show the above example after the first entry is removed. 

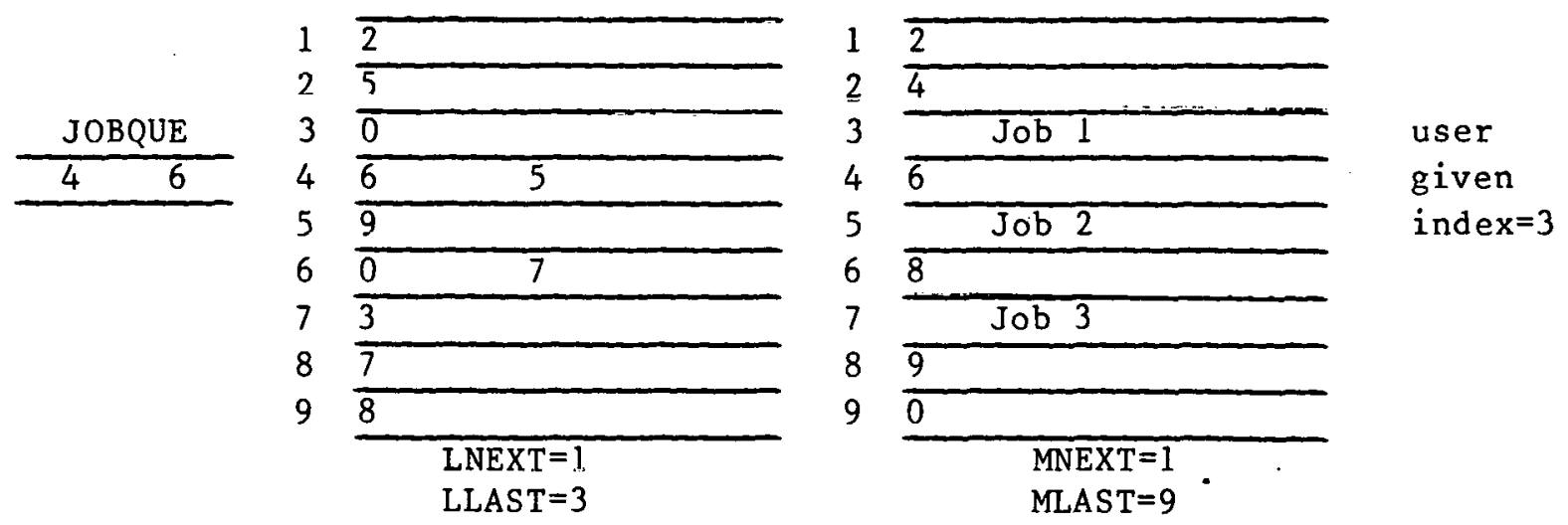

To add to a queue, the last entry is used to point to the last link (o1d link) in the list. A new 1 ink is obtained and the MTE index is placed in its second entry. The new link's index is then placed iulu llie first entry of the old link and into the second entry of the queue. The following diagram depicts the case where an Ml'E (No. 1) is ublained aud placed into JOBQUE. Notice JOB1 is currently being processed.
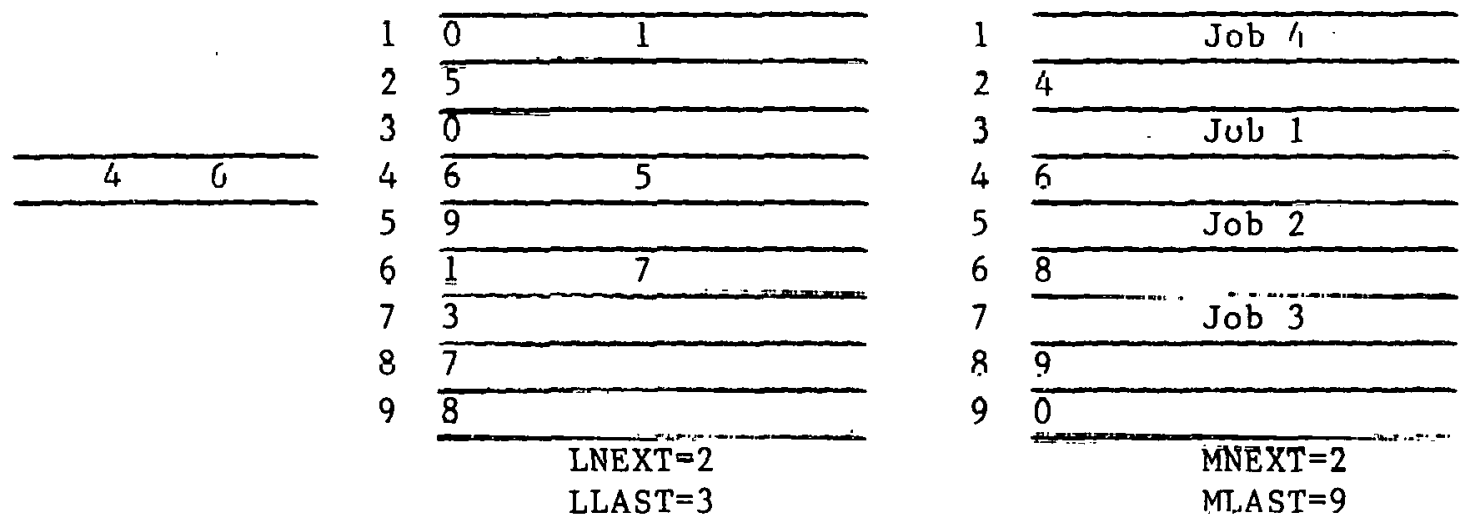

The nutlined scheme provides a flexible method of queuing jobs between software tasks. 
DISTRIBUTION :

Los Alamos Scientific Laboratory (2)

P.O. Box 1663

Los Alamos NM 87545

Attn: Dale Sparks C-9

Oak Ridge National Laboratory

P.0. Box X

Oak Ridge TN 37830

Attn: Albert Stevens ( $\mathrm{Y}-12$ Plant)

Argonne National Laboratories

9700 South Cass Avenue

Argonne IL 60439

Attn: William Lidinsky

1120 T. L. Pace

1521 J. L. Mortley

1523 B. T. Fox

2600. L. E. Hollinsworth

2610 D. C. Jones

2620 R. J. Detry

2630 E. K. Montoya

2635 P. A. Lemke

2636 W. F. Mason

2640 J. L. Tischhauser

2644 D. M. Darsey

2645 L. D. Bertholf

2648 D. H. Schroeder

2648 M. O. Vahle (2)

2648 L. F. Tolendino (48)

2650 A. D. Pepmueller

8334 R. Y. Lee

8266 E. A. Aas

3141 T. L. Werner (5)

3151 W. L. Garner (3)

For DOE/TIC (Unlimited Release)

DOE/TIC (25)

(R. P. Campbe11, 3154-3) 


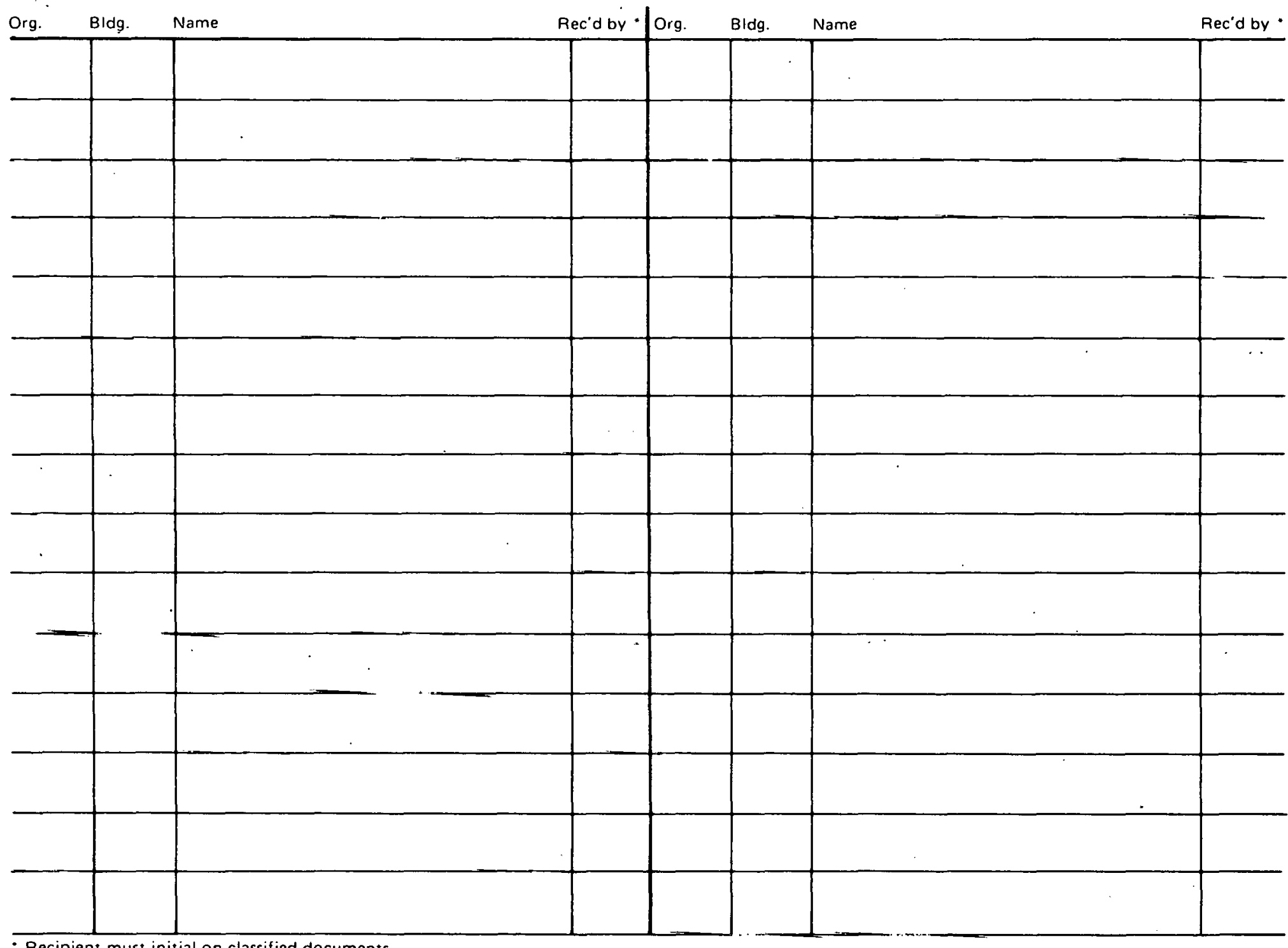

- Recipient must initial on classified documents. 